\title{
Review of: "Bma-LAD-2, an intestinal cell adhesion protein, as a potential therapeutic target for lymphatic filariasis"
}

\author{
Ramaswamy Kalyanasundaram ${ }^{1}$ \\ 1 University of Illinois at Chicago
}

Potential competing interests: The author(s) declared that no potential competing interests exist.

This is an interesting study that attempts to knock down Bma-LAD-2, a protein that is expressed on the intestinal lining of the adult female Brugia malayi, a filarial parasite of human. The study will determine the effects of knocking down on the viability of the adult worms in vitro and its ability to release the microfilariae. The study will use an SiRNA approach to knock down the Bma-LAD-2. Analysis of sera samples from infected subjects suggest that they do not carry IgE antibodies that bound to Bma-LAD-2. Thus, Bma-LAD-2 appears to be a potential therapeutic target against $B$. malayi. Overall, the concept of the study is strong, although the findings are only in vitro. Targeting the adult worms can potentially prevent the development of chronic symptoms and the fact that the microfilariae production is also reduced suggest that the approach can be potentially used to reduce the transmission of the disease as well.

Major weakness of this study is that, only in vitro data is available. Similarly, the dose of SiRNA needed to clear the worms need to be determined in a live animal. Because of the unique location of the adult parasites and the reduced flow of lymph around the parasite, it may be difficult to achieve the desired concentration of the SiRNA molecules to reach the worms. Even more challenging will be to move this to human. Bma-LAD-2 protein potentially could be a good therapeutic vaccine candidate. Although no IgE antibodies against Bma-LAD-2 were detected in the sera of infected human subjects, actual vaccination with Bma-LAD-2 will be needed to be performed to determine if the protein triggers allergic response or not. 